\title{
Management of orbital invasion in esthesioneuroblastoma: 14 years' experience
}

\author{
Ruichen Li', Shu Tian', Yi Zhu', Li Yan', Wenjia Zhu², Huatao Quan² and Shengzi Wang ${ }^{1 *}$
}

\begin{abstract}
Background: There is a scarcity of data about the prognostic value of orbital invasion in esthesioneuroblastoma (ENB), as well as about its management strategies. Indications for the preservation of orbital contents remain controversial, and the evaluation of orbital invasion has been ill defined.

Methods: This retrospective analysis contained 60 ENB patients with orbital invasion who underwent radiotherapy with or without surgery over the past 14 years. Orbital invasion was classified into three grades.

Results: There were 52 patients at stage $C$ and 8 at stage D, according to Foote classifications. Grade I, grade II and grade III orbital invasion was detected in 12, 23, and 25 patients, respectively. The median follow-up was 57 months (IQR 32-95 months). Fourteen patients received radical radiotherapy, with a 5-year overall survival (OS) of 63.5\%; 46 received surgery plus radiation, with a 5 -year OS of 70.7\%; and the difference was not statistically significant ( $p=0.847$ ). Orbital preservation was feasible in $100 \%$ of cases, including 18 cases that extended to extraocular muscles or the eye globe. Five-year locoregional relapse-free survival was 100\% in patients with prophylactic elective neck irradiation (PENI) and $58.1 \%$ in patients without PENI $(p=0.004)$. Univariate analysis showed that grade II/III orbital invasion was associated with poorer OS and progression-free survival. Neck metastasis (with a Foote stage of D) was independently associated with shorter OS and distant metastasis-free survival in multivariate analysis.

Conclusions: Our data suggested that primary radiotherapy achieved comparable survival to surgery plus radiotherapy in advanced ENB. Invasion of either the extraocular muscles or the eye globe is not a contraindication for eye-sparing surgery. Orbital invasion in grade II/III was significantly associated with adverse survival outcomes. Prophylactic radiotherapy to the neck with NO significantly reduces the risk of regional recurrence.
\end{abstract}

Keywords: Esthesioneuroblastoma, Orbital invasion, Orbital preservation, Radiotherapy, Prognostic factors

\section{Introduction}

Esthesioneuroblastoma (ENB) is a rare malignant tumor of the nasal vault that is usually diagnosed at a locally advanced stage [1]. Orbital invasion is frequently observed in ENB involving the ethmoid sinus. It has been proven that orbital invasion is associated with a poor prognosis and is commonly evaluated as stage $\mathrm{C}$ disease, according to the Kadish/Foote staging system [2]. The prognostic value of the distinction between invasion of the bony orbit and of the deeper soft tissue has been reflected in the

\footnotetext{
* Correspondence: shengziwang@fudan.edu.cn

'Department of Radiation Oncology, Eye \& ENT Hospital, Fudan University, 83 Fenyang Road, Xuhui, Shanghai 200031, People's Republic of China Full list of author information is available at the end of the article
}

American Joint Committee on Cancer (AJCC) staging categories, which divide ethmoid and maxillary tumors and differentiate the role of bone orbital wall invasion (T3) from invasion of the anterior two-thirds (T4a) and orbit apex (T4b), at least for squamous cell carcinoma and adenocarcinoma [3, 4]. However, whether the prognostic value of the degree of orbital invasion is applicable to ENB remains an open question due to the rarity of ENB.

The periorbit plays an important role against invasion and is recognized as a "landmark" of orbital exenteration over time in sinonasal tumors [5-7]. With the introduction of endoscopic surgery and advanced radiotherapy techniques [8], the indications for orbital exenteration are continuously changing. Agreement has not been reached

(c) The Author(s). 2019 Open Access This article is distributed under the terms of the Creative Commons Attribution 4.0 International License (http://creativecommons.org/licenses/by/4.0/), which permits unrestricted use, distribution, and reproduction in any medium, provided you give appropriate credit to the original author(s) and the source, provide a link to the Creative Commons license, and indicate if changes were made. The Creative Commons Public Domain Dedication waiver (http://creativecommons.org/publicdomain/zero/1.0/) applies to the data made available in this article, unless otherwise stated. 
on the degree of orbital invasion that is oncologically safe when sparing orbital contents. A recent study had showed that orbital preservation could be performed in cases of tumors not adherent to extraocular muscles [9]. However, the histology of sinonasal malignancies was extremely heterogeneous in previous studies [9-11], and the evaluation of orbital invasion was unclear [11], thus raising the risk of statistical bias. Furthermore, the indication for orbital exenteration in squamous cell carcinoma or adenocarcinoma may not be generalizable to ENB due to their differences in biological characteristic and prognosis.

We believe that orbital preservation can be achieved with the assistance of radiotherapy, even if the extraocular muscles and eye globe are involved in ENB. In the current study, we retrospectively reviewed and summarized our therapeutic strategy over the past 14 years for $60 \mathrm{ENB}$ patients with orbital invasion to clarify the specific degree of orbital invasion and to analyze its influence on survival and orbital preservation. To the best of our knowledge, this is the largest study in which treatment and prognostic factors have been assessed in patients with ENB invading the orbit.

\section{Material and methods}

\section{Patient characteristics}

Between April 2004 and August 2017, 60 ENB patients with orbital invasion received treatment at the Eye \& ENT Hospital, Fudan University, China, all of whom underwent radiotherapy. The exclusion criteria were as follows: 1. incomplete follow-up information or lost to follow-up; 2. insufficient medical history; 3 . evidence of distant metastasis; 4 . without orbital invasion; 5 . history of other cancers; 6 . receipt of palliative treatment. All of the patients underwent a radiological examination (MRI or high-resolution and contrast-enhanced CT scan or both) to evaluate the location and extent of the tumor. We classified orbital involvement into the following three grades [12]: grade I, bone wall invasion; grade II, invasion of extraconal fat; and grade III, involvement of extraocular muscles, eye globe, orbital apex, or optic nerve. All the cases were staged according to the Foote classifications (modified version of Kadish staging system). The clinical information of the study population is shown in Table 1.

\section{Treatment data}

A strategy of preoperative radiation was applied to cases of advanced tumors, for which surgical treatment is challenging in terms of performing radical surgery while preserving organs. For those cases at high risk of recurrence after surgery, including those with gross residual disease, positive surgical margins, and a Foote stage of C/D, postoperative radiation was performed. Patients with unresectable disease, a poor condition that precluded
Table 1 Clinical characteristics and treatment methods of evaluable patients

\begin{tabular}{|c|c|}
\hline Variable & No. (\%) \\
\hline Median age, yr. (range) & $54(22-77)$ \\
\hline \multicolumn{2}{|l|}{ Sex } \\
\hline Male & $44(73.3 \%)$ \\
\hline Female & $16(26.7 \%)$ \\
\hline \multicolumn{2}{|l|}{ Degree of orbital invasion ${ }^{a}$} \\
\hline Grade I & $12(20 \%)$ \\
\hline Grade II & $23(38.3 \%)$ \\
\hline Grade III & $25(41.7 \%)$ \\
\hline \multicolumn{2}{|l|}{ Foote stage } \\
\hline C & $52(86.7 \%)$ \\
\hline D & $8(13.3 \%)$ \\
\hline \multicolumn{2}{|l|}{ Lymph node classification } \\
\hline No & $52(86.7 \%)$ \\
\hline $\mathrm{N}+$ & $8(13.3 \%)$ \\
\hline \multicolumn{2}{|l|}{ Orbital symptoms } \\
\hline Yes & $41(68.3 \%)$ \\
\hline No & 19 (31.7\%) \\
\hline \multicolumn{2}{|l|}{ Treatment methods } \\
\hline Radical radiation & $14(23.3 \%)$ \\
\hline Preoperative radiation + surgery & $21(35 \%)$ \\
\hline Postoperative radiation +surgery & $25(41.7 \%)$ \\
\hline \multicolumn{2}{|l|}{ Surgical methods } \\
\hline Endoscopic group & $33(71.7 \%)$ \\
\hline Open group ${ }^{b}$ & $13(28.3 \%)$ \\
\hline \multicolumn{2}{|l|}{ Management of neck } \\
\hline PENI & $25(41.6 \%)$ \\
\hline TENI & $7(11.7 \%)$ \\
\hline END & $1(1.7 \%)$ \\
\hline No management & $27(45 \%)$ \\
\hline \multicolumn{2}{|l|}{ Type of radiotherapy } \\
\hline 3D-CRT & $34(56.7 \%)$ \\
\hline IMRT & $26(43.3 \%)$ \\
\hline \multicolumn{2}{|l|}{ Chemotherapy } \\
\hline Yes & $47(78.3 \%)$ \\
\hline No & $13(21.7 \%)$ \\
\hline
\end{tabular}

Abbreviation: 3D-CRT three-dimensional conformal radiotherapy, IMRT intensity-modulated radiotherapy, PENI prophylactic elective neck irradiation $T E N I$ therapeutic elective neck irradiation, END elective neck dissection ${ }^{a}$ Grade I, bone wall erosion; grade II, invasion of extraconal fat; grade III, involvement of extraocular muscles, eye globe, orbital apex, or optic nerve bopen group included lateral rhinotomy and craniofacial resection

major surgery or a refusal to undergo surgery were treated with radical radiation. Forty-seven patients received platinum-based chemotherapy.

All patients had a strong desire to preserve their eyes. The surgical methods were classified as either endoscopic 
or open (i.e., lateral rhinotomy or craniofacial resection). Elective neck dissection was performed on one $\mathrm{N}+$ patient. All patients were irradiated using three-dimensional conformal radiotherapy (3D-CRT) or intensity-modulated radiotherapy (IMRT). The patients were immobilized with a thermoplastic mask during the treatment. A set of CT images from the head to the clavicle (slice thickness of 2.5 $\mathrm{mm}$ for the primary lesion and $5 \mathrm{~mm}$ for the neck) was obtained for treatment planning. MRI was performed to identify intracranial extension if skull base bone invasion was revealed by CT. All plans were generated by a Treatment Planning System (ADAC Pinnacle 3, version 7.0, Varian Medical Systems, Palo Alto, CA). Determination of the gross tumor volume (GTV) was based on the results of MRI or a CT scan. The clinical target volume (CTV) was defined as the GTV plus a margin of $5-10 \mathrm{~mm}$, which included high-risk CTV and low-risk CTV. The planning target volume (PTV) had a margin of $3 \mathrm{~mm}$ (IMRT) or 5 $\mathrm{mm}$ (3D-CRT) added around the CTV, and the margin was reduced in areas where the volume was adjacent to critical normal structures. The average irradiation dose for the patients with definitive radiotherapy, preoperative radiation, and postoperative radiation was $67.66 \mathrm{~Gy}$ (63.678 Gy), 63.53 Gy (50-71.9 Gy) and 62.79 Gy (50-68.9 Gy), respectively. Seven patients with $\mathrm{N}+$ received ipsilateral therapeutic elective neck irradiation (TENI), with an average irradiation dose of $64.5 \mathrm{~Gy}$ (60-68.2 Gy); of $52 \mathrm{N0}$ patients, 25 patients were treated with prophylactic elective neck irradiation (PENI), with an average irradiation dose of $53.6 \mathrm{~Gy}$ ( $40-57 \mathrm{~Gy})$, while 27 were not. The dose constraints for organs at risk were as follows: spinal cord ( $\leq$ 45 Gy), optic nerve ( $\leq 54$ Gy), optic chiasm ( $\leq 54$ Gy), and brainstem ( $\leq 54 \mathrm{~Gy})$. Dose-volume histograms were retrieved from the planning system and used for planning evaluation. The treatment plans were normalized to cover 95\% of the PTVs with the prescribed dose.

\section{Follow-up}

The treatment efficacy was evaluated based on the results of a radiological examination and Response Evaluation Criteria in Solid Tumors (version 1.1). Adverse reactions were graded according to the scoring system of the Radiation Therapy and Oncology Group. Follow-up information was obtained from outpatient reviews and telephone interviews. The last investigation was held in August 2018, and the median follow-up duration was 57 months (IQR 32-95 months).

\section{Statistical analysis}

The Kaplan-Meier method was used to estimate the probability of locoregional relapse-free survival (LRFS), distant metastasis-free survival (DMFS), progressionfree survival (PFS), and overall survival (OS). Locoregional relapse is defined as follows: recurrence of primary site or cervical lymph node metastasis. Distant metastasis is defined as follows: metastasis outside primary site and cervical lymph node, such as lung, bone or liver. Overall survival is defined as follows: death by any cause. Progression is defined as follows: progression, recurrence, distant metastasis or death by any cause. The survival was calculated from the date of the first administration of therapy to the date of the event or last follow-up. The log-rank test and Cox hazard model (forward approach) were used in univariate and multivariate analyses, respectively. Variables with $p<0.05$ in the univariate analyses were included in the multivariate analyses. Statistical analyses were performed using SPSS, version 22 (SPSS, Chicago, IL). For all analyses, the $p$ values were two-sided, and $p<0.05$ was considered statistically significant.

\section{Results}

\section{Clinical features}

A total of 60 patients were included in this study, which consisted of 44 males (73.3\%) and 16 females (26.7\%), with a median age of 54 years (range $=22-77$ years). The basic clinical information is presented in Table 1 . Twelve patients $(20 \%)$ had grade I orbital invasion, 23 (38.3\%) had grade II orbital invasion, and 25 (41.7\%) had grade III orbital invasion. All primary tumors were located in the nasal cavity with $(n=8)$ or without lymph node metastasis $(n=52)$. According to the Foote staging system (modified version of Kadish staging system), the distribution of patients with stage $C$ and stage D ENB was $52(86.7 \%)$ and $8(13.3 \%)$, respectively. Most patients had orbital symptoms (68.3\%). We performed surgical resection with orbital preservation in 46 patients (100\%), and no patient underwent orbital exenteration.

\section{Survival outcomes in different treatment modalities}

Forty-six patients received surgery plus radiation with or without chemotherapy, whereas 14 patients received definitive radiotherapy with or without chemotherapy. As depicted in Fig. 1, there was no significant difference in the survival curves treated with or without surgery $(p=$ $0.847)$. Patients treated with surgery had a 5 -year OS of $70.7 \%$, compared to $63.5 \%$ in the nonsurgically treated patients. There was also no significant difference in 5year LRFS (66.3\% vs. $83.9 \%, p=0.407)$.

The average irradiation dose among the patients with definitive radiotherapy was $67.66 \mathrm{~Gy}$ (63.6-78 Gy). After treatment, $7(50 \%)$ patients had a complete response (CR), 6 (42.9\%) had a partial response (PR), and 1 (7.1\%) had progression of the disease (PD). Among the $7 \mathrm{pa}-$ tients with $\mathrm{CR}$, one patient died of cardiovascular disease, with a survival time of 58 months, after experiencing cervical recurrence three times; another patient died of distant metastasis, with a survival time of 



Fig. 1 Survival curves among various treatment modalities. a Overall survival; b Locoregional relapse free survival

50 months. The remaining 5 patients were disease-free and still alive at the end of follow-up. Figure 2 shows a comparison of the MRI before and after treatment in one typical case, which had invaded to extraocular muscle. To date, this patient had been followed up for 50 months with no recurrence. Among the 6 patients with PR, 3 patients showed abnormal signals of residual tumors, which reduced gradually and disappeared spontaneously during follow-up. Another 2 patients showed no progression of the disease. The remaining patient underwent additional surgery but died of brain metastasis, with a survival time of 9 months. The patient with PD died after a survival time of 3 months.

Of the 46 patients who underwent surgery, 21 cases received preoperative radiation and 25 cases received postoperative radiation. The median times between surgery and radiation in pre- and postoperative radiation subgroups were 35 days (12-78 days) and 27 days (13-43 days), respectively. The average dose was $63.53 \mathrm{~Gy}$ (50-71.9 Gy) and 62.79 Gy (50-68.9 Gy), respectively. The 5 -year OS was $79 \%$ for preoperative radiation and 58.2\% for postoperative radiation (Fig. 1), although the difference

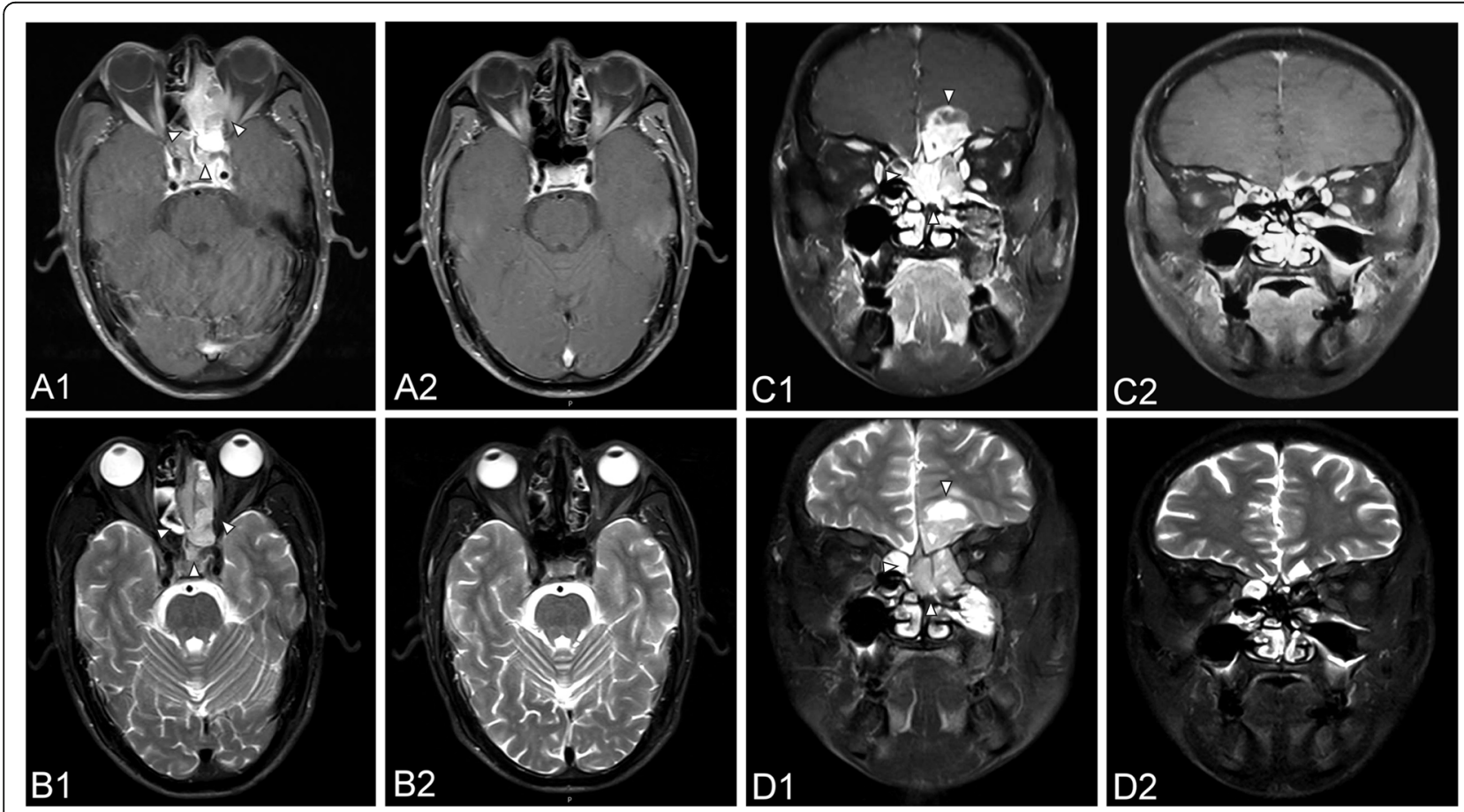

Fig. 2 Comparison of MRI before and after treatment for a 50-year-old patient with ENB (stage of C) who received definitive radiotherapy without surgery. (A) and (B) refer to axial MRI, (C) and (D) refer to coronal MRI. 1, 2 refer to MRI before treatment, after radiotherapy, respectively. MRI before treatment revealed a large mass on the left nasal cavity and ethmoid sinus, which invaded the extraocular muscle of the left orbit (A1 arrowhead, B1 arrowhead). The tumor extended to beyond the midline, left frontal sinus and sphenoid sinus, and frontal lobe (C1 arrowhead, D1 arrowhead). After radiotherapy, the patient had a complete response 
was not statistically significant $(p=0.215)$. Among the 25 patients with postoperative radiation, residual tumors were found in 19 cases (76\%) after surgery. The patients without residual tumors had a 5 -year OS of $100 \%$, which fell to $53.1 \%$ in patients with residual tumors $(p=0.187)$, and a similar relationship was shown in the 5-year PFS for the two groups $(100 \%$ vs. $42.8 \%$, respectively, $p=0.088)$. As depicted in Fig. 3, after primary surgery, a residual tumor was observed in MRI. Postoperative radiation was delivered later, and the residual tumor disappeared. To date, this patient has been followed for 37 months with no recurrence. Treatment efficacy among the preoperative radiation group was as follows: 6 cases $(28.6 \%)$ had CRs, 11 cases $(52.4 \%)$ had PRs, and 4 cases (19\%) had stable disease (SD). There was no difference in 5-year OS between CRs and non-CRs ( $80 \%$ vs. $78.3 \%, p=0.941)$, nor the 5 year LRFS $(66.7 \%$ vs. $66.3 \%, p=0.521)$. In terms of radiotherapy and different surgical methods, the average irradiation dose in endoscopic group and open group was 63.62 Gy (56-68.8 Gy) and 61.87 Gy (50-71.9 Gy) ( $p=$ $0.607)$, respectively. Of the 46 patients, 5 patients $(10.9 \%)$ experienced a local recurrence, and 8 patients (17.4\%) experienced a regional recurrence.

Of the 8 patients who had clinical/radiographic evidence of neck metastasis $(\mathrm{N}+), 7$ patients had undergone definitive radiotherapy of the neck (TENI) with no regional recurrence during the follow-up period. The remaining one, who was treated with elective neck dissection, had developed neck node recurrence 28 months after treatment and underwent neck dissection again. Of the 52 patients who were $\mathrm{N} 0$ at the time of initial treatment, 27 patients were treated without any form of PENI, while 25 patients were treated with PENI with an average irradiation dose of $53.6 \mathrm{~Gy}$ (40-57 Gy). Among the 27 cases who did not receive PENI, 8 (29.6\%) developed node failures, compared with 0 of 25 patients $(0 \%)$ who did receive PENI. The 5-year LRFS was $100 \%$ in patients with PENI and $58.1 \%$ in patients without PENI $(p=0.004)$, the 5 -year DMFS was $85.3 \%$ in patients with PENI and $85.7 \%$ in patients without PENI $(p=0.762)$, the 5-year PFS was $81.8 \%$ in patients with PENI and $50 \%$ in patients without PENI $(p=0.039)$, and the 5 -year OS was $80.5 \%$ in patients with PENI and $70.1 \%$ in patients without PENI ( $p=0.689)$ (Fig. 4).

\section{Organ preservation and adverse reactions of normal tissues}

We performed surgical resection with orbital preservation in 46 patients $(100 \%)$, including 18 cases that extended to extraocular muscles or the eye globe, and no patient underwent orbital exenteration. Figure 5 shows a comparison before and after preoperative radiation in one typical case that extended to extraocular muscle. After radiation, the tumor size shrank obviously and achieved nearly a complete response, thus demonstrating the feasibility of orbital preservation. To date, this patient has been followed for 51 months with no recurrence.

Regarding acute skin and eye reactions, most patients presented with grade 1 reactions, although grade 2 skin, mucosal, and eye reactions were observed in 3,18 , and 8 patients, respectively. No grade 3 or grade 4 reactions were observed. Late toxicity was assessed in 46 surviving patients. The most common vision-related side effect was dry eye syndrome, with an incidence of $39.1 \%$ (18 in 46 cases). With regard to visual impairment, three patients developed lateral visual loss (grade 4), and 2 patients developed grade 3 visual impairment. The causes of blindness were optic neuropathy in two patients and retinopathy in one patient. Cataract formation was successfully treated with surgery in 5 patients.

\section{Prognostic analysis}

The median time to recurrence was 19.5 months (range $=6-55$ months). During the follow-up period, 6 patients experienced local recurrences, and 9 patients experienced neck node recurrences. The 5-year LRFS was $72.5 \%$. Distant metastases were recorded in 8 patients, and the 5-year DMFS was $80.9 \%$. The univariate analysis of factors predictive of locoregional control showed that treatment without chemotherapy and nonPENI were significantly associated with reduced LRFS (Table 2). The univariate and multivariate analyses of factors predictive of DMFS showed that neck metastasis (with a Foote stage of D) were significantly correlated with adverse outcomes (hazard ratio $[\mathrm{HR}]=4.713, p=$ 0.042). (Table 3).

By the end of the follow-up, 14 patients had died, with a 5 -year OS of $69 \%$ and a 5 -year PFS of $57.7 \%$. The results of the univariate analysis showed that grade II/III orbital invasion and a Foote stage of D (neck metastasis) had significant effects on OS (Table 2). Univariate analysis showed that grade II/III orbital invasion, a Foote stage of $\mathrm{D}$ (neck metastasis), radiotherapy of 3D-CRT, and treatment without PENI were associated with reduced PFS. As depicted in Fig. 6, grade I orbital invasion was associated with better OS and PFS than was grade II/III. The 5-year OS was $100 \%$ in patients with grade I orbital invasion and $61.4 \%$ in patients with grade II/III orbital invasion $(p=0.043)$. The 5 -year OS was $72.3 \%$ for a Foote stage of C, compared to $50 \%$ for a Foote stage of D $(p=0.026)$. In the multivariate analysis, a Foote stage of $\mathrm{D}(\mathrm{HR}=4.419, p=0.013)$ was significantly predictive of shorter OS (Table 3). Radiotherapy of IMRT ( $\mathrm{HR}=0.323, p=0.043)$ was an independently significant good prognostic factor for PFS. 

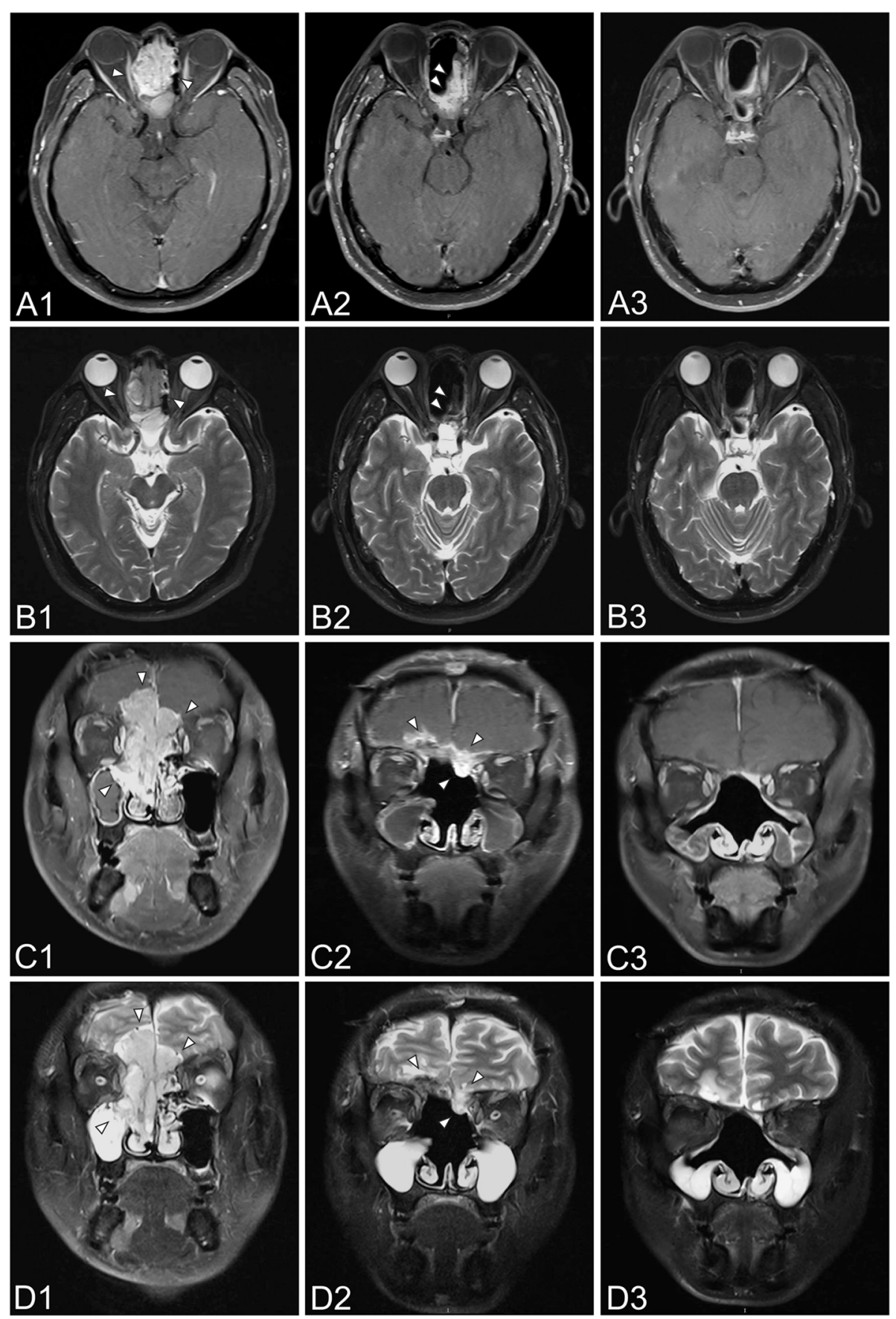

Fig. 3 Comparison of MRI before and after treatment for a 38-year-old patient with ENB (stage of C) who received surgery plus postoperative radiation. $(\mathbf{A})$ and $(\mathbf{B})$ refer to axial MRI, and $(\mathbf{C})$ and $(\mathbf{D})$ refer to coronal MRI. 1, 2, and 3 refer to MRI before treatment, after surgery, and after radiotherapy, respectively. MRI before treatment revealed a large mass on bilateral nasal cavity and ethmoid sinus, which invaded the extraconal fat of the right orbit (A1 arrowhead, B1 arrowhead). The tumor extended to right maxillary sinus and sphenoid sinus, bilateral anterior skull base and frontal lobe (C1 arrowhead, D1 arrowhead). After surgery, residual tumor was observed the site of ethmoid sinus (A2 arrowhead, B2 arrowhead), bilateral anterior skull base and frontal lobe (C2 arrowhead, D2 arrowhead). After postoperative radiation, the residual tumor disappeared

\section{Discussion}

Due to the rarity of ENB, there is limited data on this cancer in the literature. As far as we know, this is a retrospective study with the largest cohort of ENB with orbital invasion thus far within a single institution. Several interesting and new findings were discovered from 

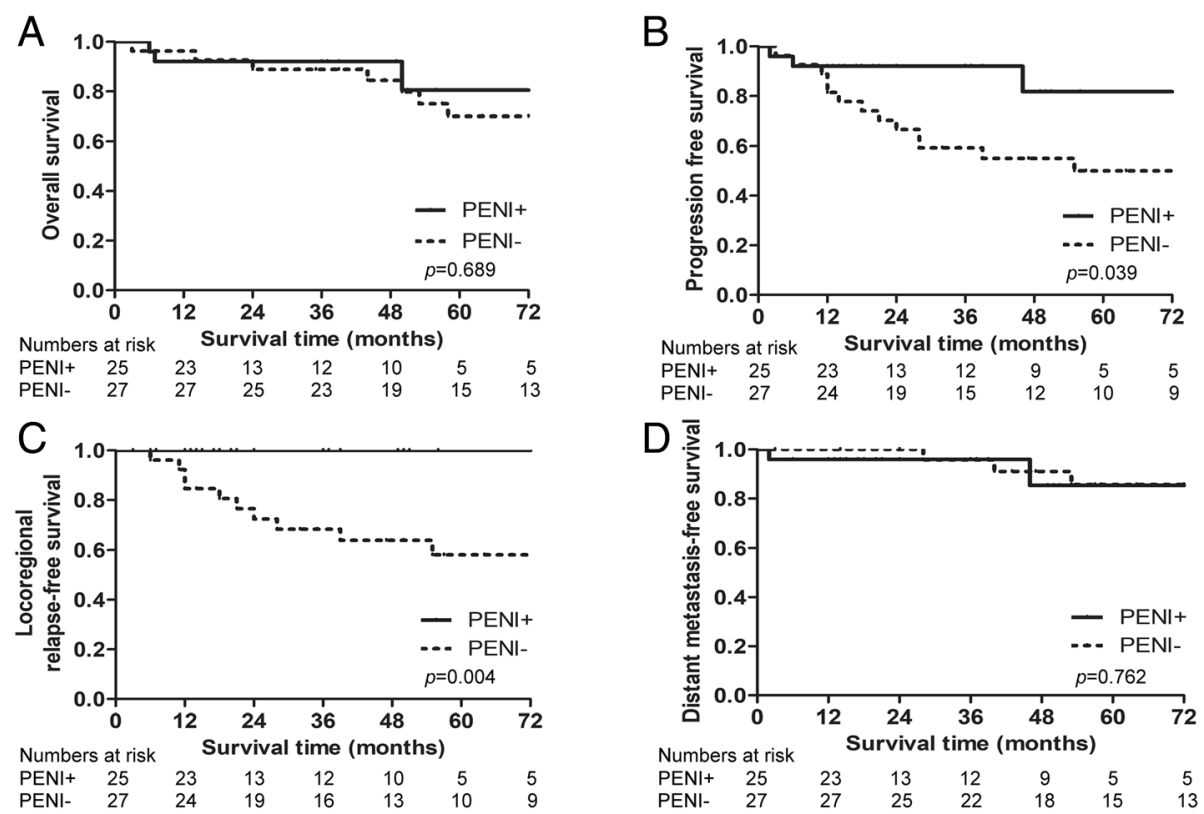

Fig. 4 Survival curves for 52 patients treated with prophylactic elective neck irradiation (PENI)/non-PENI. a Overall survival; b Progression free survival; c Locoregional relapse free survival; $\mathbf{d}$ Distant metastasis free survival

this report. First, the prognostic value of distinction between invasion of the bony orbit and deeper soft tissue was found to exist in ENB. Second, the survival rate after radical radiation was comparable to the combination of surgery and radiotherapy among patients with aggressive tumors and stages (Foote stage of C/D). Third, no patient was managed with orbital exenteration, even in cases that extended to extraocular muscles or the eye globe.

There is no standardized staging system for ENB, and many staging systems have been proposed for ENB. The Kadish staging system [2] is still the most popular system because of its simplicity and ease of application. According to the Kadish system, tumors that extend beyond the nose and paranasal sinuses are designated as stage C [2]. Therefore, the system recognizes orbital invasion as a relatively negative prognostic factor, given that tumors with orbital invasion are classified as stage C. However, there are many criticisms of the Kadish system, and the prognosis for each stage is inconsistent among studies [13] because the details of local extension of the tumor were not assessed. Joshi et al. [14] used the National Cancer Database to evaluate the prognostic ability of the Kadish staging system. This multivariate analysis controlling for stage failed to demonstrate clear survival differences between stages in the Kadish system, which poorly predicted prognosis over a 10-year horizon. The prognostic value of the distinction between invasion of the bony orbit and deeper soft tissue had been reflected in the AJCC staging categories, which divide bone orbital wall invasion (T3) from invasion of the anterior two-thirds (T4a) and orbit apex (T4b) for sinonasal tumors of epithelial origin [3, 4]. In our study, we followed the classification method proposed by Iannetti [12] (stated in Material and Methods). Using this classification method, a univariate analysis showed that grade I orbital invasion was associated with better OS and PFS than was grade II/III. The outcome means that deeper orbital invasion is a negative prognostic factor in ENB. To our surprise, there was no significant difference between grade II and grade III in survival rate, and the 5-year OS of patients with involvement of the orbital apex was comparable to that of patients without involvement $(62.3 \% \mathrm{vs}$. $70.2 \%, p=0.173$ ). Based on this observation, we believe that the Iannetti classification method could be a good reference for clinical treatment, but a long-term additional study with a larger sample size should be conducted to help determine whether orbital invasion is an independent prognosis factor in ENB.

The treatment strategy of ENB remains controversial. Due to the rarity of these tumors and the lack of prospective randomized controlled clinical trials, there is no generally accepted therapeutic regimen for ENB [15-17]. Comprehensive treatment is usually recommended, as the majority of cases present with advanced ENB, and surgical resection followed by postoperative radiotherapy demonstrated better treatment outcomes with 5-year OS rates of $60-88 \%$ in previous retrospective reports [18-20], compared to radiotherapy alone $(29-54 \%)[15,19,21,22]$. Although previous reports indicated that comprehensive treatment was superior to radical radiotherapy, the results should be interpreted with caution, because patients with 

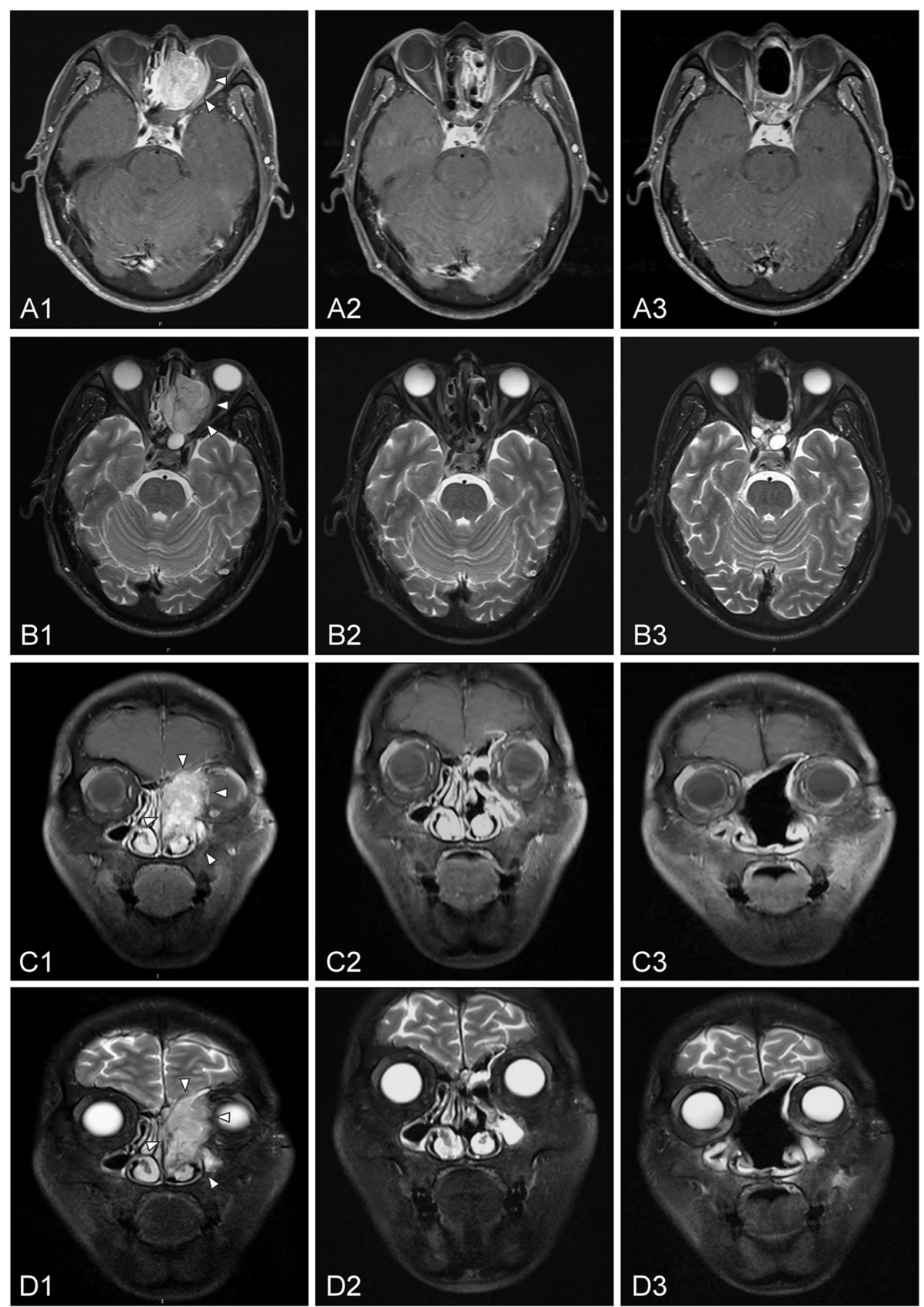

Fig. 5 Comparison of MRI before and after treatment for a 43-year-old patient with ENB (stage of C) who received preoperative radiation plus surgery. (A) and (B) refer to axial MRI, and (C) and (D) refer to coronal MRI. 1, 2, and 3 refer to MRI before treatment, after preoperative radiation, and after surgery, respectively. MRI before treatment revealed a large mass on left nasal cavity and ethmoid sinus, which invaded the extraocular muscle of the left orbit (A1 arrowhead, B1 arrowhead). The tumor extended to left anterior skull base (C1 arrowhead, D1 arrowhead)

wider infringement of lesions may be given radical radiotherapy rather than surgery. Therefore, there was a significant case selection bias between various treatment modalities $[15,19]$. The clinical data of 21 patients with T4 ENB treated with $\mathrm{C}$-ion radiotherapy were retrospectively reviewed by Suefji [23]. The 3-year overall survival and local control rate were 88.4 and $83 \%$, respectively.
Nakamura et al. [24] described 42 consecutive patients who received proton beam therapy with curative intent for ENB. Twenty-eight (67\%) patients had Kadish stage C and achieved a 5-year OS of 76\%. In our study, 7 (50\%) patients had a complete response among the definitive radiotherapy group. More interestingly, the abnormal signals of residual tumors which disappeared spontaneously 
Table 2 Univariate analysis predicting OS, PFS, LRFS, DMFS

\begin{tabular}{|c|c|c|c|c|c|c|c|c|}
\hline \multirow[t]{2}{*}{ Variable } & \multicolumn{2}{|c|}{ 5-year OS } & \multicolumn{2}{|c|}{ 5-year PFS } & \multicolumn{2}{|c|}{ 5-year LRFS } & \multicolumn{2}{|c|}{ 5-year DMFS } \\
\hline & $\%$ & $p$ & $\%$ & $p$ & $\%$ & $p$ & $\%$ & $p$ \\
\hline \multicolumn{9}{|c|}{ Age } \\
\hline$>55$ & 76.1 & 0.201 & 65 & 0.596 & 74.8 & 0.877 & 86.8 & 0.371 \\
\hline$\leq 55$ & 61.9 & & 48.8 & & 68 & & 72.7 & \\
\hline \multicolumn{9}{|c|}{ Sex } \\
\hline Male & 70.4 & 0.461 & 56.4 & 0.739 & 65.9 & 0.168 & 84 & 0.101 \\
\hline Female & 64 & & 58.3 & & 93.8 & & 70 & \\
\hline \multicolumn{9}{|c|}{ Degree of orbital invasion ${ }^{a}$} \\
\hline Grade I & 100 & $0.043^{*}$ & 91.7 & $0.041^{*}$ & 91.7 & 0.223 & 100 & 0.121 \\
\hline Grade $\|/\| \|$ & 61.4 & & 49.2 & & 66.8 & & 75.7 & \\
\hline \multicolumn{9}{|c|}{ Foote stage } \\
\hline$C$ & 72.3 & $0.026^{*}$ & 61.7 & $0.046^{*}$ & 73.9 & 0.449 & 84.6 & $0.009^{*}$ \\
\hline $\mathrm{D}$ & 50 & & 37.5 & & 64.3 & & 57.1 & \\
\hline \multicolumn{9}{|c|}{ Lymph node classification } \\
\hline NO & 72.3 & $0.026^{*}$ & 61.7 & $0.046^{*}$ & 73.9 & 0.449 & 84.6 & $0.009^{*}$ \\
\hline $\mathrm{N}+$ & 50 & & 37.5 & & 64.3 & & 57.1 & \\
\hline \multicolumn{9}{|c|}{ Invasion of orbital apex or optic nerve } \\
\hline Yes & 62.3 & 0.173 & 53 & 0.154 & 80.8 & 0.849 & 80.8 & 0.387 \\
\hline No & 70.2 & & 59.4 & & 71.4 & & 81.5 & \\
\hline \multicolumn{9}{|c|}{ Chemotherapy } \\
\hline Yes & 65.5 & 0.335 & 63.9 & 0.216 & 80.3 & $0.016^{*}$ & 78.4 & 0.372 \\
\hline No & 80.2 & & 37.9 & & 50.5 & & 87.5 & \\
\hline \multicolumn{9}{|c|}{ Surgical methods } \\
\hline Endoscopic group & 73.8 & 0.796 & 54.5 & 0.358 & 68.1 & 0.268 & 84.6 & 0.8 \\
\hline Open group ${ }^{\mathrm{b}}$ & 67.7 & & 46.2 & & 59.2 & & 80 & \\
\hline \multicolumn{9}{|c|}{ Treatment methods } \\
\hline Radical radiation & 63.5 & 0.847 & 69.3 & 0.394 & 83.9 & 0.407 & 83.1 & 0.885 \\
\hline Radiation + surgery & 70.7 & & 53.8 & & 66.3 & & 80.2 & \\
\hline \multicolumn{9}{|c|}{ Type of radiotherapy } \\
\hline 3D-CRT & 60.2 & 0.077 & 46.9 & $0.032^{*}$ & 67.7 & 0.277 & 71.6 & $0.028^{*}$ \\
\hline IMRT & 89.7 & & 79.6 & & 82.8 & & 100 & \\
\hline \multicolumn{9}{|c|}{ PENI } \\
\hline Yes & 80.5 & 0.689 & 81.8 & $0.039^{*}$ & 100 & $0.004^{*}$ & 85.3 & 0.762 \\
\hline No & 70.1 & & 50 & & 58.1 & & 85.7 & \\
\hline
\end{tabular}

Abbreviation: OS overall survival, PFS progression free survival, $L R F S$ locoregional relapse free survival, DMFS distant metastasis free survival, $3 D$-CRT threedimensional conformal radiotherapy, IMRT intensity-modulated radiotherapy, PENI prophylactic elective neck irradiation

*Statistically significant, $p<0.05$

${ }^{a}$ Grade I, bone wall erosion; grade II, invasion of extraconal fat; grade III, involvement of extraocular muscles, eye globe, orbital apex, or optic nerve

bopen group included lateral rhinotomy and craniofacial resection 
Table 3 Multivariate Cox regression analysis of OS, PFS, LRFS, DMFS

\begin{tabular}{|c|c|c|c|c|c|c|c|c|}
\hline \multirow[t]{2}{*}{ Variable } & \multicolumn{2}{|l|}{ OS } & \multicolumn{2}{|l|}{ PFS } & \multicolumn{2}{|l|}{ LRFS } & \multicolumn{2}{|l|}{ DMFS } \\
\hline & $\mathrm{HR}(95 \% \mathrm{Cl})$ & $p$ & $\mathrm{HR}(95 \% \mathrm{Cl})$ & $p$ & HR (95\% Cl) & $p$ & $\mathrm{HR}(95 \% \mathrm{Cl})$ & $p$ \\
\hline Degree of orbital invasion ${ }^{a}$ & & & & & Not included & & Not included & \\
\hline Grade II/III vs. Grade I & - & 0.971 & - & 0.076 & & & & \\
\hline Foote stage & & & & & Not included & & & \\
\hline D vs. C & $4.419(1.362-14.345)$ & $0.013^{*}$ & - & 0.07 & & & $4.713(1.059-20.98)$ & $0.042^{*}$ \\
\hline Chemotherapy & Not included & & Not included & & & & Not included & \\
\hline No vs. Yes & & & & & $3.519(1.133-10.926)$ & $0.03^{*}$ & & \\
\hline Type of radiotherapy & Not included & & & & Not included & & & \\
\hline IMRT vs. 3D-CRT & & & $0.323(0.108-0.963)$ & $0.043^{*}$ & & & - & 0.945 \\
\hline
\end{tabular}

Abbreviation: OS overall survival, PFS progression free survival, LRFS locoregional relapse free survival, DMFS distant metastasis free survival, $H R$ hazard ratio, $C I$ confidence interval, $3 D$-CRT three-dimensional conformal radiotherapy, IMRT intensity-modulated radiotherapy

*Statistically significant, $p<0.05$

aGrade I, bone wall erosion; grade II, invasion of extraconal fat; grade III, involvement of extraocular muscles, eye globe, orbital apex, or optic nerve

during follow-up were found in 3 patients with PR. Radical radiotherapy still achieved a 5-year OS of 63.5\%, which was higher than that of surgery plus postoperative radiation (58.2\%). These results suggest that radical radiotherapy is not merely palliative but has therapeutic efficacy as a primary treatment for a notable percentage of locally advanced or unresectable ENBs.

For Kadish/Foote stage $\mathrm{C}$ tumors, there is little consensus regarding the timing of radiotherapy for a combination of surgery and radiation. The complex anatomical location, as well as visual and facial structures, is "challenging" in terms of performing radical surgery while preserving functional organs and improving local control rates. Only a few studies have been reported in terms of preoperative radiation in the treatment of sinonasal cancers. Erik et al. [25] reported 79 patients with sinonasal cancer who were treated with preoperative radiation, and orbital exenteration was performed in $7 \%$ of these patients. A total of 11,160 sinonasal malignancy patients were identified from the National Cancer Data Base for analysis by Robin [26]. The findings were validated in propensity score matching, and it is important to note that neoadjuvant chemoradiotherapy was associated with achieving a negative surgical margin and improved OS. For ENB, Polin et al. [27] showed significant tumor reduction in two-thirds of patients treated with preoperative
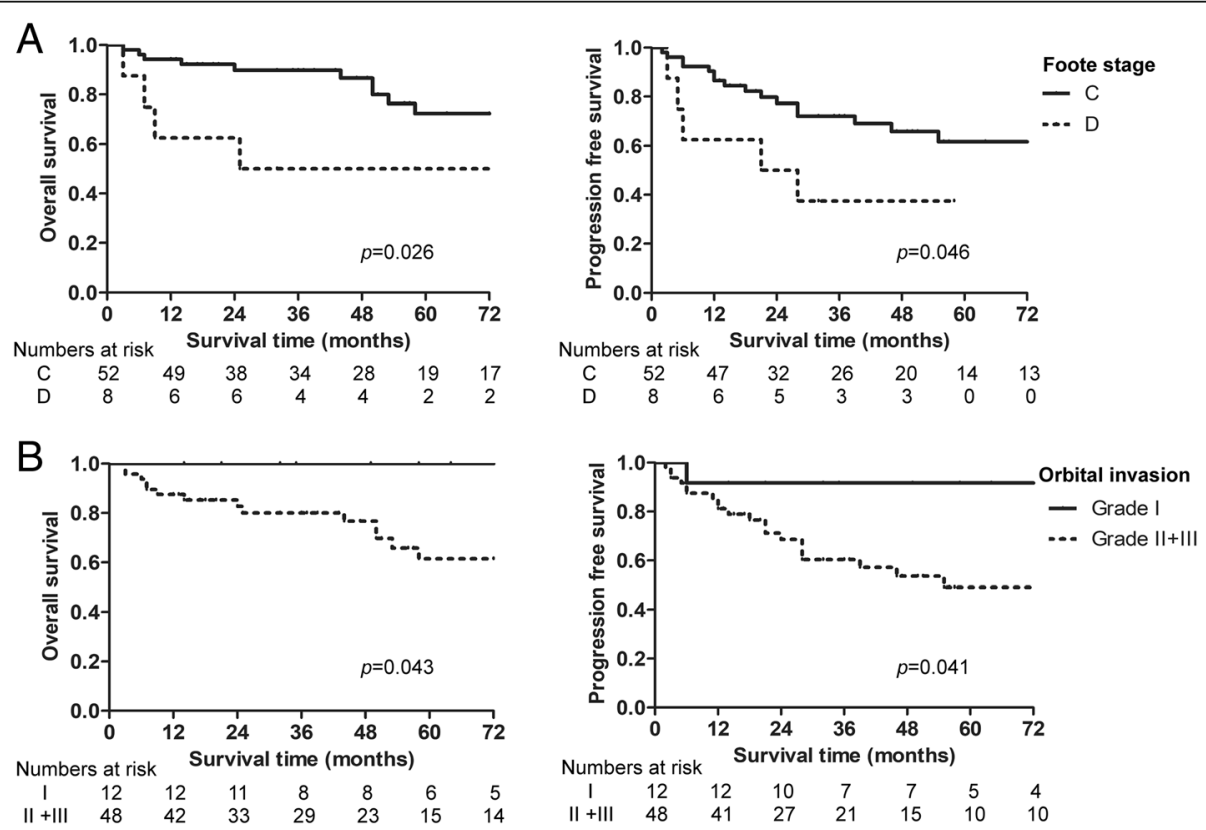

Fig. 6 Kaplan-Meier OS (left) and PFS (right) curves for patients with ENB stratified by various clinical factors. a Survival curves for patients stratified by Foote stage; $\mathbf{b}$ for patients stratified by orbital invasion 
radiation with or without chemotherapy and increased recurrence-free survival in those responders. Sohrabi et al. [28] reported complete pathologic responses in two stage C ENB patients treated with neoadjuvant concurrent radiochemotherapy. In our series, preoperative radiotherapy yielded the most promising results, including a higher long-term survival, than did postoperative radiotherapy (5-year OS $79 \%$ for preoperative radiation vs. $58.2 \%$ for postoperative radiation, $p=0.215$ ). Considering the high residual rate of primary surgery $(76 \%)$, we believe that preoperative radiation not only improves the tumor resection rate but also increases the potential for orbital preservation surgery by reducing tumor mass and eliminating microscopic lesions at the periphery of the tumor. The treatment strategy of preoperative radiotherapy and/or chemotherapy followed by surgery for Kadish stage C/D disease was also advocated for by a European position paper on endoscopic management of tumors of the nose, paranasal sinuses and skull [29].

The indications for orbital exenteration are continuously changing, from invasion of orbital periosteum [30] to beyond the periosteum [6], to extraconal fat [10], and extraconal muscles [9]. Imola and Schramm [10] reported 66 patients in which the orbit was preserved, even in cases of limited involvement of extraconal fat. Orbital exenteration was performed in 12 cases, and similar survival rates were achieved in cases of orbital preservation and exenteration. In a cohort reported on by Lisan et al. [9], orbital preservation was undertaken in $66 \%$ of cases, whereas orbital exenteration was performed in cases of invasion of the extraocular muscles, ocular globe, or orbital apex. Local control rates and 5-year survival were similar in both groups. In previous studies, which include several pathological types [9-11, 25] with varying prognoses, the evaluation of orbital invasion was ill defined $[11,25]$. In our study, the histology was limited to ENB. Due to the good response to radiotherapy, no patient underwent orbital exenteration. The 5-year OS was 69\% among all cases, which is consistent with the rates of $65-75 \%$ reported in previous studies [18-20]. These results suggested that orbital preservation did not lead to poor survival rate, and invasion of extraocular muscles or the eye globe was not a contraindication for eye-sparing surgery in ENB.

In our study, lymph node status is the important prognostic factor affecting OS, DMFS, and PFS. Regarding 8 patients with $\mathrm{N}+, 7$ patients underwent elective neck irradiation with no regional recurrence. According to this observation, we consider that patients in stage D disease with LN metastasis require only irradiation to the neck instead of neck dissection. Regarding patients with N0, ENB has a high rate of late regional recurrence (20$44 \%)[31,32]$. However, the value of prophylactic elective neck irradiation (PENI) for patients with NO neck status is still unclear. In a large cohort (116 patients) reported by Yin et al. [33], PENI was an independent favorable predictor for regional controlling, and the author recommended PENI as a part of initial treatment strategy for patients with Foote stage B/C tumors. Two other studies hold a differing opinion that PENI plays a limited role in improving regional control [34, 35]. Our study showed that PENI improved the LRFS significantly from 58.1 to $100 \%(p=0.004)$. Although the $p$-value was not statistically significant, the 5 -year OS was still higher in patients treated with PENI than patients not treated with PENI.

Although patients who received chemotherapy had better LRFS compared to those who received treatment without chemotherapy in our study, the therapeutic effects of chemotherapy on ENB cannot be determined for the reason that the cohort lacked a standard chemotherapy protocol that was uniformly applied. Further clinical studies are needed to evaluate the value of chemotherapy for ENB. Our study had several other limitations. It was a retrospective analysis based on data from a single center. The Hyams grading system [36] that can be used as a prognostic indicator for ENB was not assessed in our study. A multicenter clinical trial is indispensable for further investigation.

\section{Conclusion}

Our data suggest that orbital invasion in grade II/III was significantly associated with adverse survival outcomes. Invasion of either the extraocular muscles or eye globe is not a contraindication for eye-sparing surgery. Primary radiotherapy achieved comparable survival to surgery plus radiotherapy in advanced and unresectable ENB. Prophylactic radiotherapy to the neck in patients with N0 significantly reduces the risk of regional recurrence.

\section{Abbreviations \\ 3D-CRT: Three-dimensional conformal radiotherapy; AJCC: American Joint Committee on Cancer; CR: Complete response; CTV: Clinical target volume; DMFS: Distant metastasis-free survival; ENB: Esthesioneuroblastoma; GTV: Gross tumor volume; HR: Hazard ratio; IMRT: Intensity-modulated radiotherapy; LRFS: Locoregional relapse-free survival; OS: Overall survival; PD: Progression disease; PENI: Prophylactic elective neck irradiation; PFS: Progression-free survival; PR: Partial response; PTV: Planning target volume; SD: Stable disease; TENI: Therapeutic elective neck irradiation}

\section{Acknowledgements}

Not applicable.

\section{Authors' contributions}

$\mathrm{RCL}$ and SZW provided the conception and design of this study; RCL, WJZ, and HTQ collected the patient data; RCL, ST, YZ, and LY analyzed and interpreted the patient data; RCL and ST have been involved in drafting the manuscript; $Y Z$, LY, and SZW have been involved in revising it critically for important intellectual content. All authors read and approved the final manuscript.

\section{Funding}

This study was supported by CSCO-Merck Serono Cancer Research Fund [grant number: Y-MT 2015-037]. 


\section{Availability of data and materials}

The datasets used and/or analysed during the current study are available from the corresponding author on reasonable request.

\section{Ethics approval and consent to participate}

The study was approved by the Clinical Research Ethics Committee of the Eye \& ENT Hospital of Fudan University. All procedures performed in studies involving human participants were in accordance with the ethical standards of the institutional and/or national research committee and with the World Medical Association Declaration of Helsinki (version 2002) and the additional requirements. Informed consent was obtained from all individual participants included in the study.

\section{Consent for publication}

Written informed consent for publication of their clinical details and/or clinical images was obtained from the patient.

\section{Competing interests}

The authors declare that they have no competing interests.

\section{Author details}

'Department of Radiation Oncology, Eye \& ENT Hospital, Fudan University, 83 Fenyang Road, Xuhui, Shanghai 200031, People's Republic of China. ${ }^{2}$ Department of E.N.T, Eye \& ENT Hospital, Fudan University, Shanghai 200031, China.

Received: 14 February 2019 Accepted: 29 May 2019

Published online: 13 June 2019

\section{References}

1. Abdelmeguid AS. Olfactory Neuroblastoma. Curr Oncol Rep. 2018;20:7.

2. Kadish S, Goodman M, Wang CC. Olfactory neuroblastoma. A clinical analysis of 17 cases. Cancer. 1976;37:1571-6.

3. Llorente JL, Lopez F, Suarez C, Hermsen MA. Sinonasal carcinoma: clinical, pathological, genetic and therapeutic advances. Nat Rev Clin Oncol. 2014; 11:460-72.

4. Cantu G, Solero CL, Mariani L, Mattavelli F, Pizzi N, Licitra L. A new classification for malignant tumors involving the anterior skull base. Arch Otolaryngol Head Neck Surg. 1999;125:1252-7.

5. Lund VJ, Howard DJ, Wei WI, Cheesman AD. Craniofacial resection for tumors of the nasal cavity and paranasal sinuses--a 17-year experience. Head Neck. 1998:20:97-105.

6. Suarez C, Ferlito A, Lund VJ, Silver CE, Fagan JJ, Rodrigo JP, et al. Management of the orbit in malignant sinonasal tumors. Head Neck. 2008; 30:242-50.

7. Muscatello L, Fortunato S, Seccia V, Marchetti M, Lenzi R. The implications of orbital invasion in sinonasal tract malignancies. Orbit. 2016;35:278-84.

8. Orlandi E, Giandini T, lannacone E, De Ponti E, Carrara M, Mongioj V, et al. Radiotherapy for unresectable sinonasal cancers: dosimetric comparison of intensity modulated radiation therapy with coplanar and non-coplanar volumetric modulated arc therapy. Radiother Oncol. 2014;113:260-6.

9. Lisan Q, Kolb F, Temam S, Tao Y, Janot F, Moya-Plana A. Management of orbital invasion in sinonasal malignancies. Head Neck. 2016;38:1650-6.

10. Imola MJ, Schramm VJ. Orbital preservation in surgical management of sinonasal malignancy. Laryngoscope. 2002;112:1357-65.

11. Safi AF, Behn L, Rothamel D, Guntinas-Lichius O, Beutner D, Nickenig HJ, et al. Therapy of sinonasal malignancies invading the orbit-orbital exenteration versus preservation of the orbit plus radiotherapy. J Craniomaxillofac Surg. 2017:45:258-61.

12. lannetti G, Valentini V, Rinna C, Ventucci E, Marianetti TM. Ethmoido-orbital tumors: our experience. J Craniofac Surg. 2005;16:1085-91.

13. Konuthula N, Iloreta AM, Miles B, Rhome R, Ozbek U, Genden EM, et al. Prognostic significance of Kadish staging in esthesioneuroblastoma: an analysis of the National Cancer Database. Head Neck. 2017;39:1962-8.

14. Joshi RR, Husain Q, Roman BR, Cracchiolo J, Yu Y, Tsai J, et al. Comparing Kadish, TNM, and the modified Dulguerov staging systems for esthesioneuroblastoma. J Surg Oncol. 2019;119:130-42.

15. Dulguerov P, Allal AS, Calcaterra TC. Esthesioneuroblastoma: a meta-analysis and review. Lancet Oncol. 2001;2:683-90.
16. Platek ME, Merzianu M, Mashtare TL, Popat SR, Rigual NR, Warren GW, et al. Improved survival following surgery and radiation therapy for olfactory neuroblastoma: analysis of the SEER database. Radiat Oncol. 2011;6:41.

17. Su SY, Bell D, Ferrarotto R, Phan J, Roberts D, Kupferman ME, et al. Outcomes for olfactory neuroblastoma treated with induction chemotherapy. Head Neck. 2017;39:1671-9.

18. Tajudeen BA, Arshi A, Suh JD, Palma-Diaz MF, Bergsneider M, Abemayor E, et al. Esthesioneuroblastoma: an update on the UCLA experience, 20022013. J Neurol Surg B Skull Base. 2015;76:43-9.

19. De Bonnecaze G, Lepage B, Rimmer J, Al HA, Vairel B, Serrano E, et al. Longterm carcinologic results of advanced esthesioneuroblastoma: a systematic review. Eur Arch Otorhinolaryngol. 2016;273:21-6.

20. Ow TJ, Hanna EY, Roberts DB, Levine NB, El-Naggar AK, Rosenthal DI, et al Optimization of long-term outcomes for patients with esthesioneuroblastoma. Head Neck. 2014;36:524-30.

21. Kane AJ, Sughrue ME, Rutkowski MJ, Aranda D, Mills SA, Buencamino R, et al. Posttreatment prognosis of patients with esthesioneuroblastoma. J Neurosurg. 2010;113:340-51

22. Ozsahin M, Gruber G, Olszyk O, Karakoyun-Celik O, Pehlivan B, Azria D, et al. Outcome and prognostic factors in olfactory neuroblastoma: a rare cancer network study. Int J Radiat Oncol Biol Phys. 2010;78:992-7.

23. Suefuji H, Koto M, Demizu Y, Saitoh Jl, Shioyama Y, Tsuji H, et al. A retrospective multicenter study of carbon ion radiotherapy for locally advanced olfactory neuroblastomas. Anticancer Res. 2018:38:1665-70.

24. Nakamura N, Zenda S, Tahara M, Okano S, Hayashi R, Hojo H, et al. Proton beam therapy for olfactory neuroblastoma. Radiother Oncol. 2017;122:368-72.

25. Fernstrom E, Nyman J, Hammerlid E, Holmberg E, Haugen-Cange $H_{\text {, }}$ Petruson K, et al. Results of preoperative chemoradiotherapy for patients with advanced cancer of the nasal cavity and paranasal sinuses. Acta Otolaryngol. 2017;137:1292-300.

26. Robin TP, Jones BL, Gordon OM, Phan A, Abbott D, McDermott JD, et al. A comprehensive comparative analysis of treatment modalities for sinonasal malignancies. Cancer. 2017;123:3040-9.

27. Polin RS, Sheehan JP, Chenelle AG, Munoz E, Larner J, Phillips CD, et al. The role of preoperative adjuvant treatment in the management of esthesioneuroblastoma: the University of Virginia experience. Neurosurgery. 1998;42:1029-37.

28. Sohrabi S, Drabick JJ, Crist H, Goldenberg D, Sheehan JM, Mackley HB. Neoadjuvant concurrent chemoradiation for advanced esthesioneuroblastoma: a case series and review of the literature. J Clin Oncol. 2011;29:e358-61.

29. Lund VJ, Stammberger H, Nicolai P, Castelnuovo P, Beal T, Beham A, et al. European position paper on endoscopic management of tumours of the nose, paranasal sinuses and skull base. Rhinol Suppl. 2010;22:1-143.

30. Larson DL, Christ JE, Jesse RH. Preservation of the orbital contents in cancer of the maxillary sinus. Arch Otolaryngol. 1982;108:370-2.

31. Monroe AT, Hinerman RW, Amdur RJ, Morris CG, Mendenhall WM. Radiation therapy for esthesioneuroblastoma: rationale for elective neck irradiation. Head Neck. 2003;25:529-34.

32. Demiroz C, Gutfeld O, Aboziada M, Brown D, Marentette LJ, Eisbruch A. Esthesioneuroblastoma: is there a need for elective neck treatment? Int J Radiat Oncol Biol Phys. 2011;81:e255-61.

33. Yin ZZ, Luo JW, Gao L, Yi JL, Huang XD, Qu Y, et al. Spread patterns of lymph nodes and the value of elective neck irradiation for esthesioneuroblastoma. Radiother Oncol. 2015;117:328-32.

34. Zafereo ME, Fakhri S, Prayson R, Batra PS, Lee J, Lanza DC, et al. Esthesioneuroblastoma: 25 -year experience at a single institution. Otolaryngol Head Neck Surg. 2008;138:452-8.

35. Noh OK, Lee SW, Yoon SM, Kim SB, Kim SY, Kim CJ, et al. Radiotherapy for esthesioneuroblastoma: is elective nodal irradiation warranted in the multimodality treatment approach? Int J Radiat Oncol Biol Phys. 2011;79:443-9.

36. Bell D, Saade R, Roberts D, Ow TJ, Kupferman M, DeMonte F, et al. Prognostic utility of Hyams histological grading and Kadish-Morita staging systems for esthesioneuroblastoma outcomes. Head Neck Pathol. 2015;9:51-9.

\section{Publisher's Note}

Springer Nature remains neutral with regard to jurisdictional claims in published maps and institutional affiliations. 